\title{
DEVELOPMENT OF SOFT-HARDWARE PLATFORM FOR TRAINING SYSTEM DESIGN OF ELECTROTECHNICAL COMPLEXES AND ELECTRIC DRIVES
}

\author{
Ekaterina A. Koltunova ${ }^{1}$, Kseniya V. Ikonnikova ${ }^{1 *}$, and Sergei V. Lyapushkin ${ }^{1}$ \\ ${ }^{1}$ Tomsk Polytechnic University, 634050, Tomsk, Russia
}

\begin{abstract}
The article presents the results of the development of software and hardware platform as the equipment for the training of children and youth work skills with robotics, allowing in the future to apply this knowledge in practice, implementing automation system for home use. We consider the problems of existing solutions. The main difference is the integration of the proposed fees and extensions into a single set by connecting the connectors and the ability to connect third-party components from different manufacturers, without limiting users. As well as a simplified method using a visual object-oriented programming allows you to immediately engage in the work. Prepared lessons and tasks in the game style simplifies the information and allows you to understand how you can apply one or another technical solution.
\end{abstract}

\section{Introduction}

Automation of any manufacturing systems and processes is a key point of technology development [1-2]. Complication of applicable methods demand increasing number of specialists, therefore fast adaptation in studying of any subject helps accelerate the adoption of new information and development in terms of career [3-4]. One, familiar with initial knowledge base in electronics and laws of robotics, introspects deeper in nuances and have no need in extra time for explanation of main theories of physics and electrical engineering. Therefore, a new training tool for the electromechanical systems is needed.

\section{Concept of the kit}

The most successful way of information flow on circuit design is a kit (usually laboratory works in training stands given in that form). It is necessary to create a variant of kit with open architecture and accessories, which can allow construct robotics systems for carrying out development work in domestic conditions, to solve educational and scientific tasks and to automate scientific experiments. The kit should solve these problems with a view to

*Corresponding author: $\underline{\text { kkonna@tpu.ru }}$ 
minimizing the time for its development and acceptable price for mass consumer. And also be able for consistent complication of works in progress.

The concept of that kit built on the basis of the following abstracts:

1. Functional extensibility through the use of additional modules;

2. The ability of organizing a network of several controllers;

3. Establish communication between controller and modern gadgets (smartphone, tablet PC etc.) through support of communication protocols;

4. The ability of uploading an applications for using standard procedures (navigation, audio and video processing, management of complex electromechanical objects);

5. The ability of creating software for controller by user with minimal level of qualification in the field of programming by using graphical programming languages and providing a templates to address specific tasks (for example AC motor control, synchronization of multi-axis motion systems, etc.);

6. The ability of equipping the controllers with additional modules, made by third-party (including user himself) to address the unique challenges.

\section{Experimental results}

The resulting work is software-hardware platform "MexConStructor" - equipment for schools and youth centers of innovation creation, and for private use.

The kit consists of set of PCBs, several mechanical platforms, sensors, wires and application. Developed set of PCBs with microprocessor STM32F4 (Figure 1) allows user to conveniently use the entire periphery, and has a variety of options. To this was realizable, connectors for different types of interfaces (CAN, I2C, RS-485 etc.) and analog and digital inputs and outputs are applied. A special feature is the expansion-PCB for Arduino-components, which allows connect everything that required for work (Figure 1). Kit designed in one style, allowing user to collect the necessary PCBs in one robotic system.

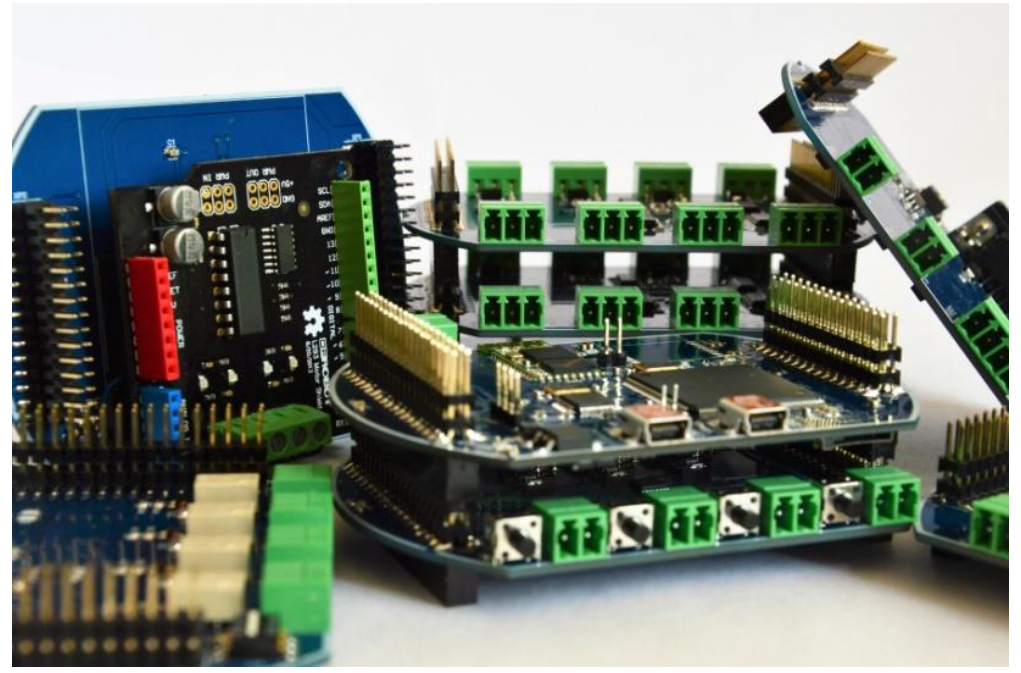

Fig. 1. Expansion-PCBs.

Proposed a set of sensors and mechanics are widely available and commonly used. Users, who already have their own components with any interface, can adapt it for work (Figure 2) 


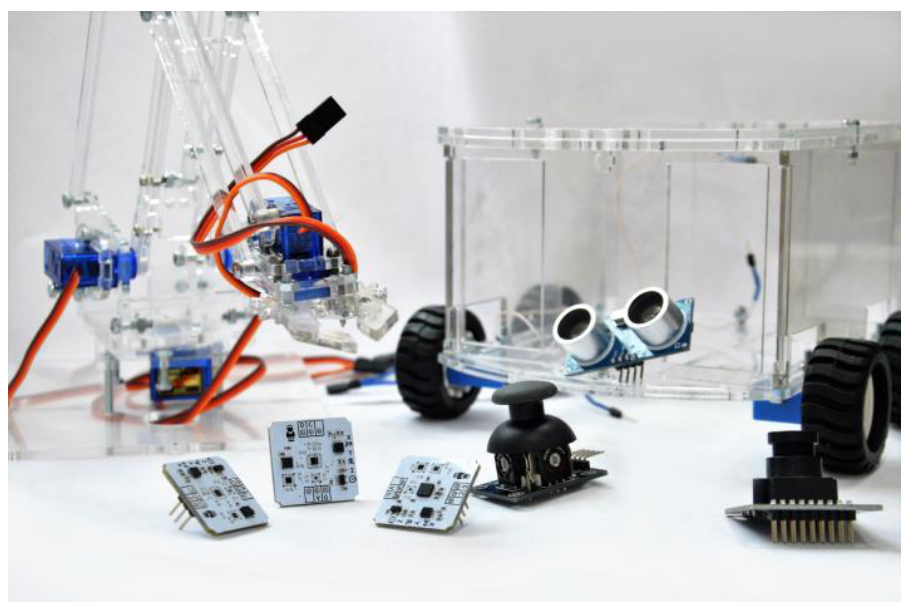

Fig. 2. Electronic components.

The main competitors of the product is the Arduino platform, robotic systems WEX, LEGO Mindstorm and analogues. Unlike competing technologies, the proposed solution makes it possible to work directly with digital devices without complicated stage communications settings.

\section{Conclusion}

In addition to that, the kit must provide the control for household appliances (including hipower), get access via standard Internet tools, set the logic by graphic and text programming languages (with the possibility of easy learning and subsequent complication of programs while operating the equipment).

Cost characteristics are at the level Arduino series controllers and significantly less than robotic kits. Unlike competing products, in the price included the system of a graphical programming MexBIOS, allowing user to create the project firmware simultaneously in 5 languages (with the choice of language by the user for each single project solved subtasks) [5]. The use of such software allows users with minimal knowledge in programming quite easily find a way to create the desired robotic complex programs (followed by the development of other ways of creating programs with increasing complexity and detail tasks.

The platform is open both in terms of hardware implementation and software. The openness of the platform should lead to the emergence of numerous applications precedents with an opportunity to copy the most successful options for the use of third-party users. Mass use ensured by the ease of mastering a specialized programming language MexBIOS, which allows graphical methods and due to built in ready-made templates, to create complex control algorithms easily enough for unskilled users. A training course in the form of a game with increasing complexity is created for children and adolescents. Successful proposed ideas are applicable in practice.

\section{References}

1. N. Gusev, S. Lyapushkina, M. Kovalenko, Integrated automation of technological processes (Publisher of Tomsk Polytechnic University, Tomsk, 2011) 
2. N. Gusev, S. Lyapushkina, M. Kovalenko, Automation of technological complexes and systems in the industry (Publisher of Tomsk Polytechnic University, Tomsk, 2011)

3. K. Ikonnikova, L. Ikonnikova, E. Koltunova, Turkish Online Journal of Educational Technology, 557 (2015)

4. K. Ikonnikova, L. Ikonnikova, E. Koltunova, Turkish Online Journal of Educational Technology, 488 (2015)

5. N. Gusev, Bulletin of South Ural State University. Series: Energy, 15, 59 (2015) 